Revista Arbitrada Interdisciplinaria KOINONIA

Año V. Vol V. N9. Enero - Junio 2020

Hecho el depósito de Ley: FA2016000010 ISSN: 2542-3088

FUNDACIÓN KOINONIA (F.K). Santa Ana de Coro. Venezuela.

Diego Javier Bastidas-Logroño; Oscar Omar Espíndola-Lara; Alex Darío Palma-Rivera

http://dx.doi.org/10.35381/r.k.v5i9.648

Implementación del bootstrap como una metodología ágil en la web

Bootstrap implementation as an agile methodology on the web

\author{
Diego Javier Bastidas-Logroño \\ info@sistemasdj.com \\ Instituto Tecnológico Superior Tsáchila, Santo Domingo \\ Ecuador \\ https://orcid.org/0000-0003-3924-7468 \\ Oscar Omar Espíndola-Lara \\ oespindola@msn.com \\ Universidad Nacional De Chimborazo, Riobamba \\ Ecuador \\ https://orcid.org/0000-0002-1364-1363 \\ Alex Darío Palma-Rivera \\ dalfp@outlook.es \\ Instituto Tecnológico Superior Tsáchila, Santo Domingo \\ Ecuador \\ https://orcid.org/0000-0002-0646-0185
}

Recibido: 25 de octubre de 2019

Revisado: 20 de noviembre de 2019

Aprobado: 20 de diciembre de 2019

Publicado: 20 de enero de 2020

\title{
RESUMEN
}

La investigación tiene como objetivo general proponer el Bootstrap como una metodología ágil en principiantes de desarrollo web como un medio de supervivencia. Metodológicamente, se llevó a cabo por medio de una metodología descriptiva, apoyada en modalidad de proyecto factible, aplicándose un instrumento tipo cuestionario de escala dicotómica con 21 alternativas de respuestas, el cual se procedió a realizar una prueba piloto para conocer su confiabilidad, mediante el coeficiente de Kuder Richardson 20, teniéndose como resultado 0,89 . El uso del desarrollo web en la actualidad está en auge, debido a esto, se ha pensado en elaborar una metodología ágil con bootstrap en la web ya que los medios informáticos no solo nos sirven para un desarrollo cognitivo, sino también para un nivel apropiado de vida. Con este conocimiento se podrá mantener un medio de vida al entender y aplicar esta metodología de desarrollo de aplicaciones web. 
Revista Arbitrada Interdisciplinaria KOINONIA

Año V. Vol V. N9. Enero - Junio 2020

Hecho el depósito de Ley: FA2016000010

ISSN: 2542-3088

FUNDACIÓN KOINONIA (F.K). Santa Ana de Coro. Venezuela.

Diego Javier Bastidas-Logroño; Oscar Omar Espíndola-Lara; Alex Darío Palma-Rivera

Descriptores: Software de código abierto; programa de ordenador; diseño de sistemas. (Palabras tomadas del Tesauro UNESCO).

\begin{abstract}
The general objective of the research is to propose Bootstrap as an agile methodology in web development beginners as a means of survival. Methodologically, it was carried out through a descriptive methodology, supported by a feasible project modality, applying a dichotomous questionnaire type instrument with 21 response alternatives, which proceeded to conduct a pilot test to determine its reliability, using the Kuder Richardson coefficient 20, and resulting in 0.89 . The use of web development is currently booming, due to this, it has been thought to develop an agile methodology with bootstrap on the web since computer media not only serve us for cognitive development, but also for an appropriate level of life. With this knowledge, a livelihood can be maintained by understanding and applying this web application development methodology.
\end{abstract}

Descriptors: Open source software; computer software, systems design. (Words taken from the UNESCO Thesaurus).

\title{
INTRODUCCIÓN
}

La investigación busca mostrar como el desarrollo web con software libre, bootstrap y su almacenamiento en un hosting con sus diferentes característicaspodrá tener un medio de supervivencia en las familias. Para realizar el análisis y desarrollar el diseño de web segura es necesario conocer los detalles y características de los entornos de desarrollo enfocados al software libre subyacentes. A su vez, deberán analizarse los servicios que se proporcionan un hosting, y sus detalles de funcionamiento. En el presente trabajo se ha optado por dar un enfoque eminentemente técnico, comenzando por aplicar características básicas del desarrollo web con software libre o sin licencias. La información existente hoy en día, tanto en la bibliografía tradicional (libros, revistas, artículos técnicos.) como en el propio Internet (páginas Web, listas de correo, grupos de noticias.) respecto al tema genérico abordado en este documento, "El uso del Bootstrap como un medio de autosuficiencia para las familias del mundo" así como las vulnerabilidades y protecciones asociadas, es realmente extensa, y cualquier 
informático podrá indagar en este apasionante mundo del desarrollo web y subida a un host. .

Bootstrap como un conjunto de herramientas, que se representa por estar disponible en código abierto desde el año 2011, es un factor clave en cuanto a la creación de contenidos web. Se ha convertido en el proyecto de mayor éxito entre los muchos disponibles en GitHub(El software que opera GitHub fue escrito en Ruby onRails.) y no es raro que por ello sea utilizado por multitud de entidades. Incluso organizaciones como la NASA recurren a sus funciones y facilidad de implementación. Para el desarrollo web simplemente se necesita un IDE (entorno de desarrollo integrado), en esta investigación usaremos Dreamweaver. Para que un usuario pueda manejar y mejorar su economía y se convierta en un ser autosuficiente se requiere:

1. Un IDE y/o editor

2. Un computador

3. Servicio de Internet.

Este trabajo está dirigido particularmente a toda la gente que es capaz de leer y manejar una computadora para que con estos conocimientos adquiridos en esta obra pueda mejorar su economía y la de sus semejantes.A continuación, se plantea el siguiente objetivo General: "Proponer el Bootstrap como una metodología ágil en principiantes de desarrollo web como un medio supervivencia".

En la actualidad el no uso de las nuevas tecnologías de la información y comunicación como un medio de autosuficiencia sería algo ilógico, y es imperativo que el mundo conozca que es sencillo aplicar métodos básicos de diseño web y almacenamiento en un hosting Linux un desarrollo simple de Font end y back end, y de esta manera tener medios para subsistir y generar empleo en este mundo competitivo. 


\section{METODOLOGÍA DE INVESTIGACIÓN}

La investigación se fundamentó desde una perspectiva cuantitativa descriptiva, apoyada en modalidad de proyecto factible con la finalidad de presentar el Bootstrap como una alternativa para el manejo de la economía familiar y de pequeños emprendimientos que permiten promover una mejor calidad de vida, la modalidad factible es explicada por la Universidad Pedagógica Experimental Libertador (2012), como:

El proyecto que consiste en la investigación, elaboración y desarrollo de una propuesta de un modelo operativo viable para solucionar problemas, requerimientos o necesidades de organización o grupos sociales; puede referirse a la formulación de políticas, programas, tecnologías, métodos o procesos (p. 23).

En este sentido, para conocer la necesidad y viabilidad de la propuesta sobre el Bootstrap, se procedió a aplicar un instrumento tipo cuestionario de escala dicotómica con 21 alternativas de respuestas, el cual se procedió a realizar una prueba piloto para conocer su confiabilidad, mediante el coeficiente de Kuder Richardson 20, teniéndose como resultado 0,89 lo cual lo cataloga como altamente confiable para su aplicación.

De ese modo se procedió a trabajar con la técnica de la encuesta a la población conformada por las personas que poseen computadoras en la ciudad de Santo Domingo de los Tsáchilas - Ecuador, la cual se encuentra conformada por 270876 Usuarios, (Censo 2010), aplicándose la el cálculo de la muestra poblacional del siguiente modo:

$1-\alpha=95 \%$

$\mathrm{Z}=1,96$

$\mathrm{E}=3 \%$

$\mathrm{E}=0,03$

$\mathrm{P}=0,5$

$P+q=1$

$Q=1-p$

$Q=1-0,5$ 
$\mathrm{Q}=0,5$

\section{Entonces:}

$\mathrm{N}=270876$ Usuarios

$\mathrm{Z}=1,96$

$E=0.03$

$\mathrm{P}=0,5$

$\mathrm{Q}=0,5$

$\mathrm{No}=Z^{2} \cdot \mathrm{P} \cdot \mathrm{Q} / \mathrm{E}^{2}$

$\mathrm{No}=(1,96)^{2} \cdot 0,5 \cdot 0,5 / 0,03^{2}$

$\mathrm{No}=3,84.0,25 / 0,0009$

$\mathrm{No}=0,96 / 0,0009$

$\mathrm{No}=1067$

\section{Entonces:}

$\mathrm{N}^{\prime}=\mathrm{No} \quad 1+(\mathrm{No}-1) / \mathrm{N}$

$N^{\prime}=1067 \quad 1+(1067-1) / 270875$

$N^{\prime}=1063$

Quiere decir que la encuesta se aplicó a 1063 usuarios.

Una vez conocida el tamaño de la muestra poblacional, se procedió a aplicar la encuesta, posteriormente la misma fue tabulada mediante la estadística descriptiva con la finalidad de describir la percepción de los encuestados en razón de conocer las fortalezas y debilidades en cuanto al Bootstrap, estructurándose los resultados de la investigación. 
Revista Arbitrada Interdisciplinaria KOINONIA

Año V. Vol V. N9. Enero - Junio 2020

Hecho el depósito de Ley: FA2016000010

ISSN: 2542-3088

FUNDACIÓN KOINONIA (F.K). Santa Ana de Coro. Venezuela.

Diego Javier Bastidas-Logroño; Oscar Omar Espíndola-Lara; Alex Darío Palma-Rivera

\section{RESULTADOS}

\section{Grafico 1}

¿Por motivos de seguridad le gustaría a Usted tener un medio, autosuficiencia utilizando las TICS?

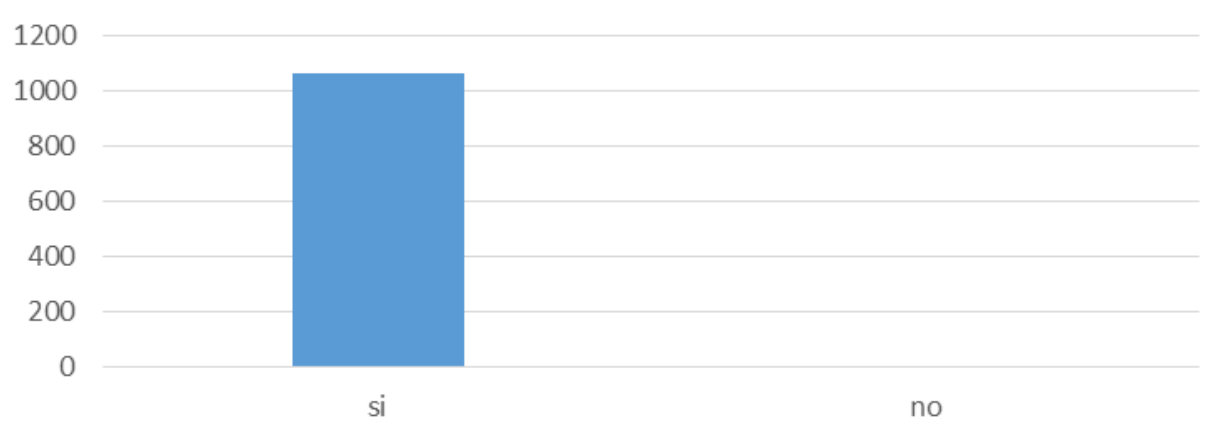

Fuente: Diego Javier Bastidas Logroño, Oscar Espíndola, Darío Palma

El 100\% de las personas encuestadas (1063 usuarios), considera que la metodología que se pretende aplicar les servirá como un medio de supervivencia.

\section{Grafico 2}

¿Por qué razón emplearía el Bootstrap?

Por una estabilidad económica

Por actualizar mis conocimientos

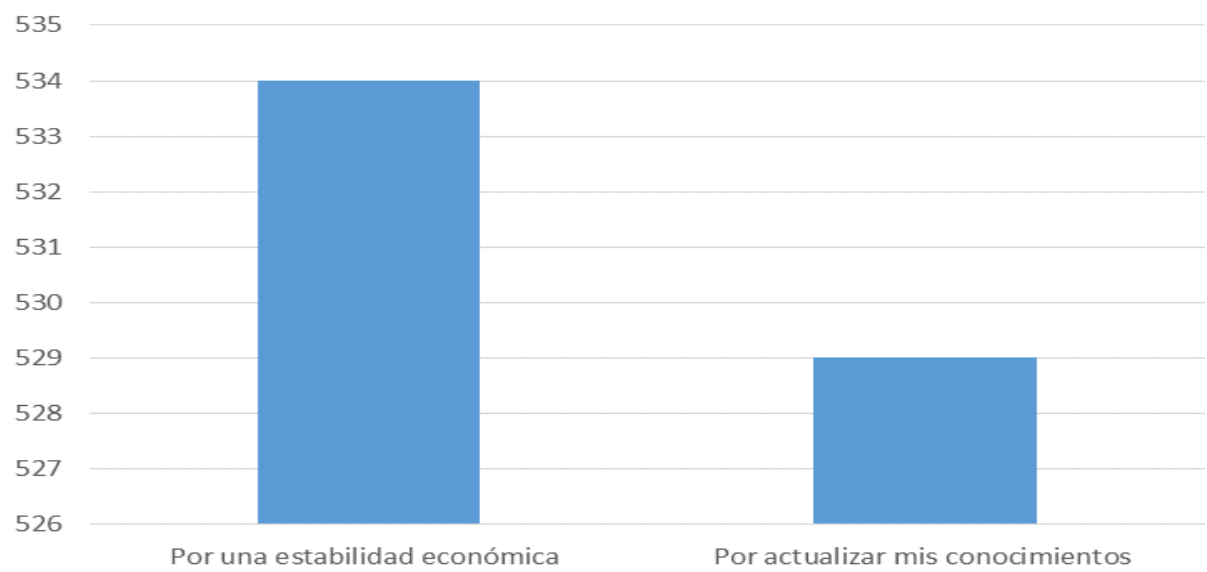

Fuente: Diego Javier Bastidas Logroño, Oscar Espíndola, Darío Palma 
Revista Arbitrada Interdisciplinaria KOINONIA

Año V. Vol V. N9. Enero - Junio 2020

Hecho el depósito de Ley: FA2016000010

ISSN: 2542-3088

FUNDACIÓN KOINONIA (F.K). Santa Ana de Coro. Venezuela.

Diego Javier Bastidas-Logroño; Oscar Omar Espíndola-Lara; Alex Darío Palma-Rivera

El 50.2\% (534 Usuarios) del personal encuestado considera que la implementación del Bootstrap mejoraría su autosuficiencia y mejorará le dará una estabilidad económica, el 49.8\% (529 Usuarios) considera que la que la implementación del Bootstrap mejoraría sus conocimientos de las nuevas tecnologías de la información y comunicación.

\section{Grafico 3}

¿Porque es importante ser autosuficiente empleando las NTICS?

Para actualizarme y tener un medio de subsistencia

Para desarrollarme como individuo

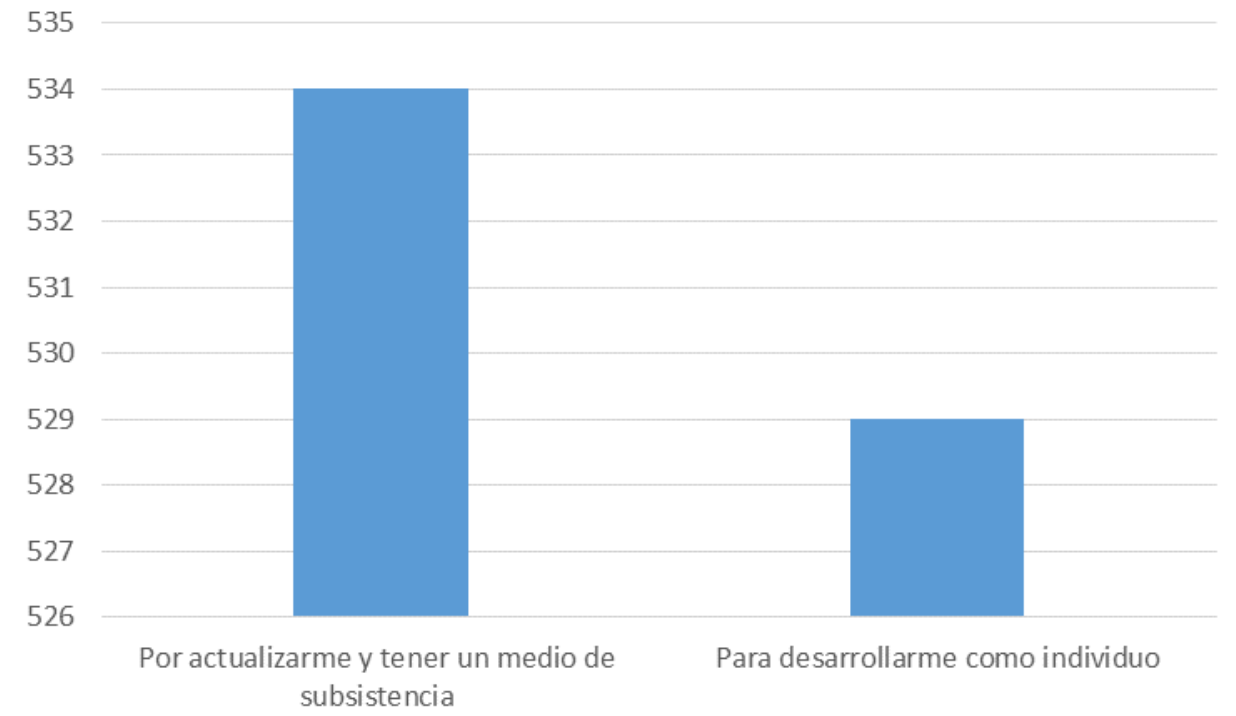

Fuente: Diego Javier Bastidas Logroño, Oscar Espíndola, Darío Palma

El 50.2\% (534 Usuarios) del personal encuestado considera que la implementación del bootstrap mejoraría su desempeño en la actualidad actualizando sus conocimientos tecnológicos y de esta manera ser autosuficiente, el 49,8\% (529 Usuarios) considera que la implementación del bootstrap y subida a un host mejoraría su desempeño obteniendo un desarrollo personal con conocimientos técnicos informáticos. 
Revista Arbitrada Interdisciplinaria KOINONIA

Año V. Vol V. No9. Enero - Junio 2020

Hecho el depósito de Ley: FA2016000010

ISSN: 2542-3088

FUNDACIÓN KOINONIA (F.K). Santa Ana de Coro. Venezuela.

Diego Javier Bastidas-Logroño; Oscar Omar Espíndola-Lara; Alex Darío Palma-Rivera

\section{Grafico 4}

¿Qué beneficios observaría para su vida el implementar dicha metodología ágil en principiantes de desarrollo web como un medio de supervivencia y generación de empleo?

Mantenerme actualizado con las NTICS y tenerlo como un ingreso extra

Mantener un estilo de vida construyendo mi propio negocio implementando las NTICS

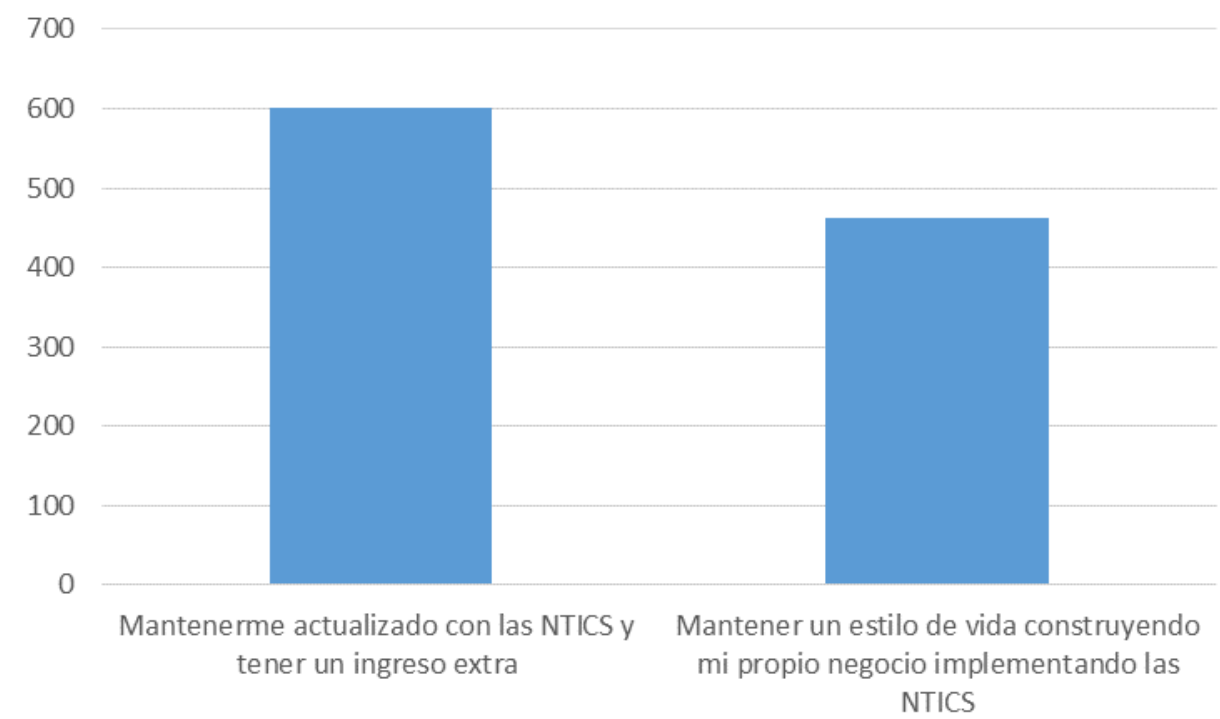

Fuente: Diego Javier Bastidas Logroño, Oscar Espíndola, Darío Palma.

El 56.4\% (600 Usuarios) del personal encuestado considera que la implementación del bootstrap les mantendrá actualizados con las NTICS y tenerlo como un ingreso extra.EI 43.6\% (463 Usuarios) considera que la implementación de esta metodología ágil les permitirá construir su propio negocio implementando las NTICS

\section{Grafico 5}

¿Le gustaría implementar esta metodología?

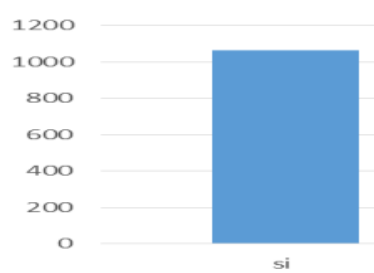

Fuente: Diego Javier Bastidas Logroño, Oscar Espíndola, Darío Palma 
SI: 1063 Usuarios $\quad$ No: 0 Usuarios No está seguro: 0 Usuarios

El 100\% del personal encuestado considera que es menester implementar este tipo de metodología como un medio de autosuficiencia y generación de empleo. Mejoramiento absoluta (100\%). La implementación como un medio de autosuficiencia fiable para software libre (Linux)como para Windows muestran un incremento de $100 \%$ de la actividad investigativa en la población.

Alto grado de mejoramiento (80\% - 99\%).- \%).- La implementación de este tipo de metodología de desarrollo web fiable tanto para Linux como para Windows con todas sus características presento plataformas y tecnologías que son compatibles con varios sistemas operativos que tienen que ser revisados antes de iniciar una implementación práctica.

Mejoramiento baja $(50 \%$ - 59\%).- las plataformas y tecnologías utilizadas son compatibles más que en ciertos aspectos con los nuevos IDEs en prueba como con Windows.

\section{RESULTADOS}

En un entorno de desarrollo del pensamiento crítico. Una vez que descargamos cualquier plantilla de entorno de desarrollo web podremos configurar en la ruta correcta del gestor de Mysql, para luego poder configurar el entorno de desarrollo integrado en este caso Dreamweaver, ya que el lector es libre de usar cualquier IDE, para luego configurar el FTP de acuerdo con las claves que se han creado en un servicio reseller o revendedor de cpanel WHM de host, con un usuario y clave sub creados para sus clientes o pymes. La calidad del software que se desarrolla debe ser libre de errores por eso es recomendable trabajar en línea con el host. Tanto las revisiones como las pruebas examinan una o más de las siguientes dimensiones de calidad del software.

Debemos tener en cuenta los siguientes parámetros:

El contenido se evalúa tanto en el nivel sintáctico como en el semántico. En el primero, se valora vocabulario, puntuación y gramática para documentos basados en texto. En el 
segundo, se valora la corrección (de la información presentada), la consistencia (a través de todo el objeto de contenido y de los objetos relacionados) y la falta de ambigüedad.

\section{Discusión}

En el contexto se verifica que existen diversas herramientas de software para el desarrollo web, sin embargo hemos adoptado este topo de desarrollo de este tipo de metodología con herramientas fiables para Windows y Linux, claro para cualquier lector y persona que desee aplicarla en su vida con un editor de texto que es Dreamweaver (o un notepad), Un servicio de host en Centos con base de datos con apache y editor gráfico.

\section{CONCLUSIONES}

El entorno de nuestra sociedad ha permitido a las personas a actualizar sin conocimientos en las NTICS por tal razón se observa un interés en aplicar las mismas para ganar dinero.

En temas de desarrollo de sitios web con una metodología ágil permitirá a los usuarios tener un amor y deseo innato de trabajar cada día en la misma permitiendo desarrollarse como persona.

La implementación de esta metodología como un medio de autosuficiencia permitirá a los mismos servir de una mejor manera a la sociedad en la que vivan.

\section{FINANCIAMIENTO}

No monetarios

\section{AGRADECIMIENTO}

Al Instituto Tecnológico Superior Tsáchila, por el apoyo técnico y científico realizado a la presente investigación. 
Revista Arbitrada Interdisciplinaria KOINONIA

Año V. Vol V. N9. Enero - Junio 2020

Hecho el depósito de Ley: FA2016000010

ISSN: 2542-3088

FUNDACIÓN KOINONIA (F.K). Santa Ana de Coro. Venezuela.

Diego Javier Bastidas-Logroño; Oscar Omar Espíndola-Lara; Alex Darío Palma-Rivera

\section{ANEXOS}

\section{Bootstrap}

Es una biblioteca que es de uso de varias plataformas o conjunto de herramientas de código abierto para diseño de sitios web y aplicaciones web. Es un Framework que fue usado por twitter para su negocio.

\section{Código Abierto}

Licencia apache año 2011

\section{Figura1}

Distribución básica bootstrap

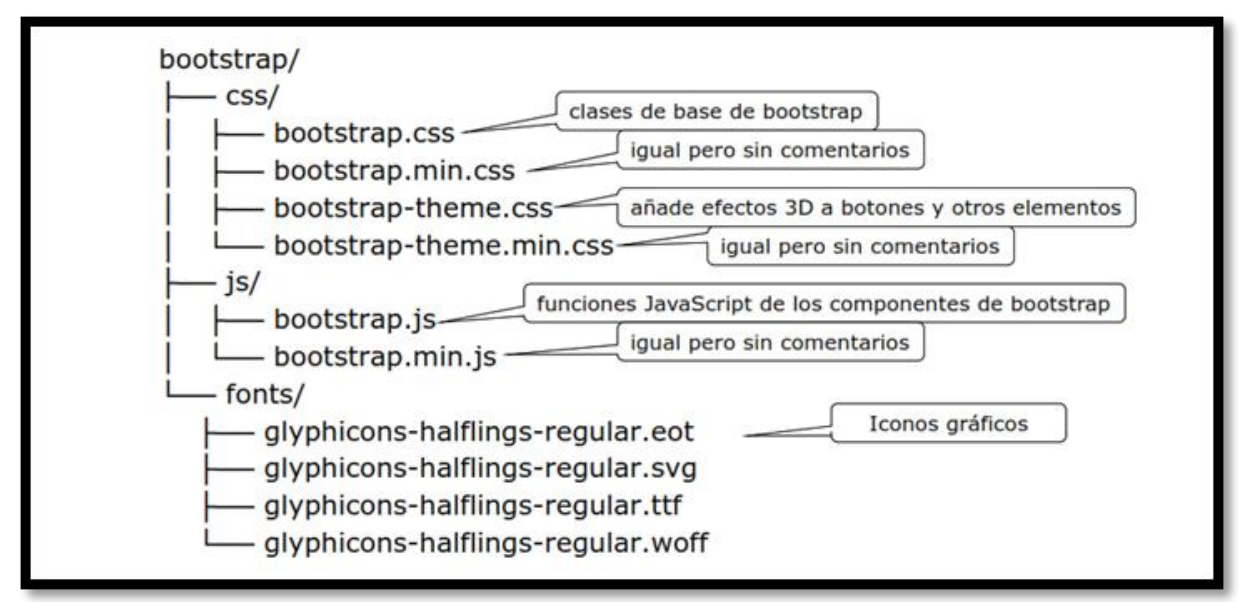

Fuente: J. Spurlock. Bootstrap. O'Reilly (2013)

\section{Responsive Web Design}

Es imperativo que el usuario visualice el entorno de desarrollo como usuario final tanto para dispositivos móviles como para la web.

\section{Bootstrap 3, Mobile First}

Una referencia con un sistema grid fluido que escala a 12 columnas al aumentar el tamaño del viewport es inédito que se revisen este tipo de innovaciones. 
Revista Arbitrada Interdisciplinaria KOINONIA

Año V. Vol V. №9. Enero - Junio 2020

Hecho el depósito de Ley: FA2016000010

ISSN: 2542-3088

FUNDACIÓN KOINONIA (F.K). Santa Ana de Coro. Venezuela.

Diego Javier Bastidas-Logroño; Oscar Omar Espíndola-Lara; Alex Darío Palma-Rivera

\section{Figura 2}

PlantillaBásica

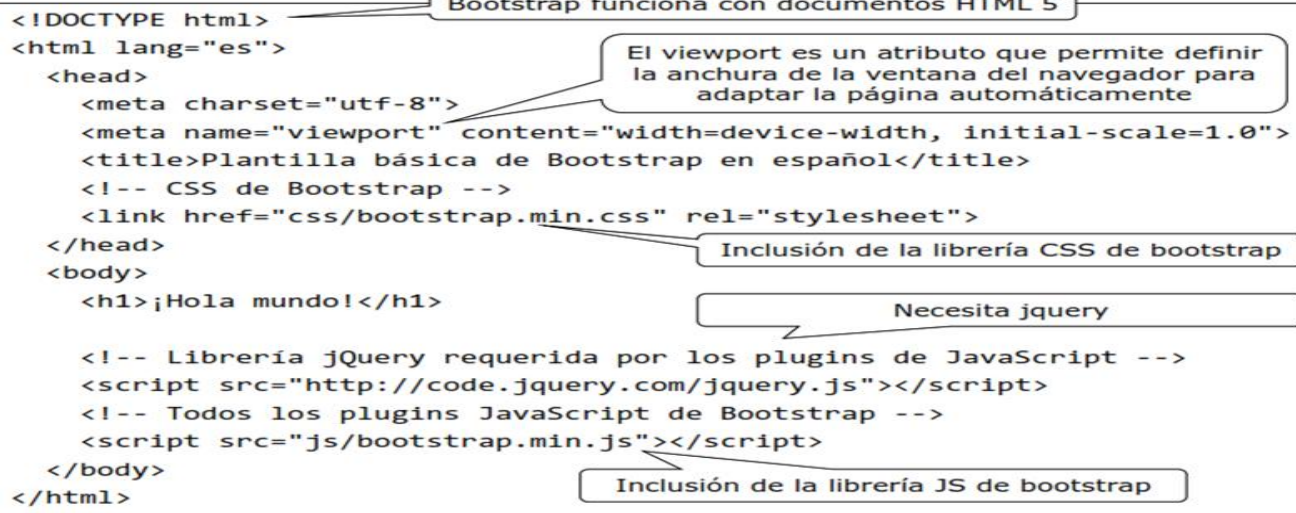

Fuente: D. Cochran. Twitter Bootstrap Web Development How-To. Packt Publishing (2012).

\section{Ejercicios Con Bootstrap}

Descargar una plantilla de bootstrap pre diseñadas y adaptarla a la empresa que vas a vender tu sitio web, es muy factible ya que es de código abierto y adaptable con una GUI profesional de código abierto.

\section{Figura 3}

Plantillas descargables adaptables gratuitas de bootstrap

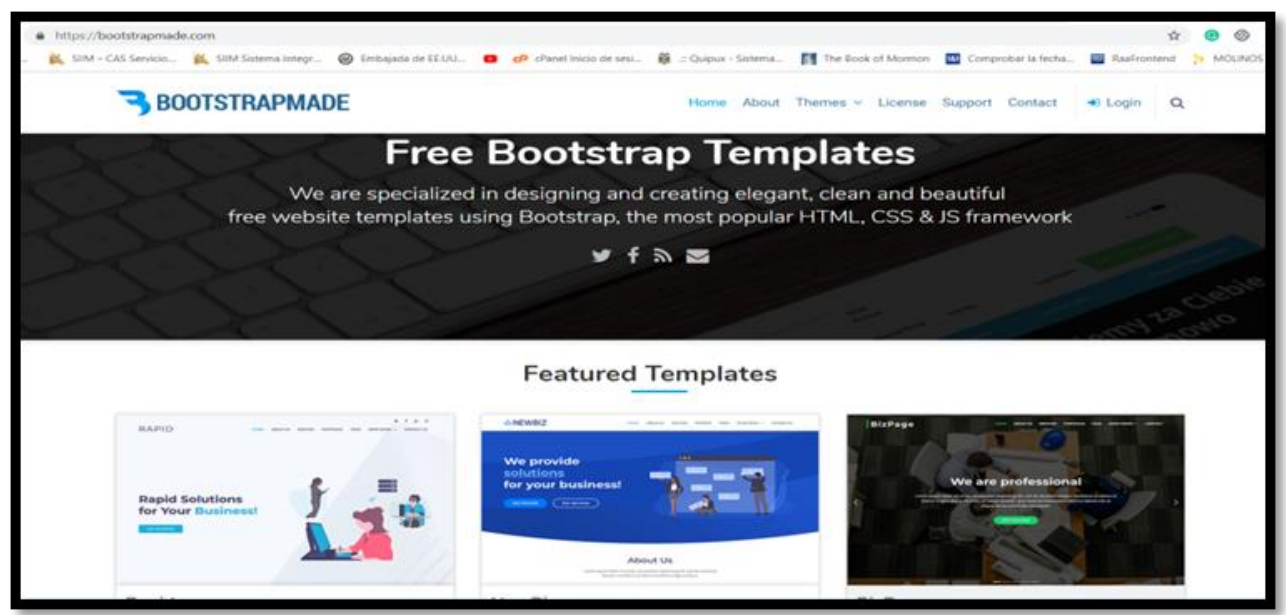

Fuente: https://bootstrapmade.com/ (2019) 
Revista Arbitrada Interdisciplinaria KOINONIA

Año V. Vol V. N9. Enero - Junio 2020

Hecho el depósito de Ley: FA2016000010

ISSN: 2542-3088

FUNDACIÓN KOINONIA (F.K). Santa Ana de Coro. Venezuela.

Diego Javier Bastidas-Logroño; Oscar Omar Espíndola-Lara; Alex Darío Palma-Rivera

\section{Wamp Server Como Gestor De Base De Datos}

WAMP es el acrónimo que nos permite gestionar php, apache, simular un servidor local, mysql.

Al mantener un servidor WAMP para la subida de archivos web y pruebas de servidor local(localhost) nos permitirá trabajar en un servidor local $o$, por lo general en un servidor Linux están configurados también para trabajar en línea con un dominio web.

Por lo general se deja la configuración de instalación del wapm server en su máquina en la dirección C:Iwamplwww y dentro de éste se deben poner los archivos descargables a modificar de bootstrap, el enlace de descarga de wampserver es: http://www.wampserver.es/.

\section{Figura 4}

Wamp server local

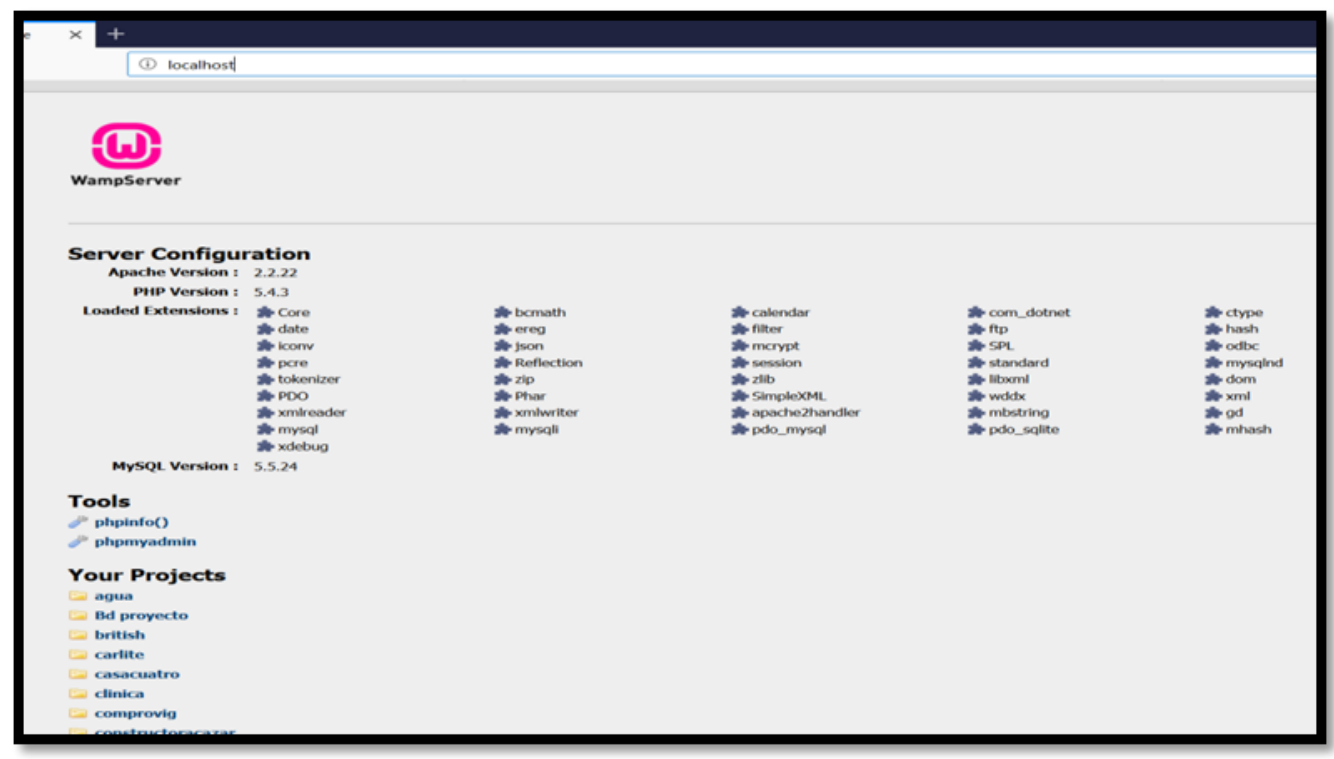

Fuente: Diego Bastida (2019) 
Revista Arbitrada Interdisciplinaria KOINONIA

Año V. Vol V. N9. Enero - Junio 2020

Hecho el depósito de Ley: FA2016000010

ISSN: 2542-3088

FUNDACIÓN KOINONIA (F.K). Santa Ana de Coro. Venezuela.

Diego Javier Bastidas-Logroño; Oscar Omar Espíndola-Lara; Alex Darío Palma-Rivera

\section{Dreamweaver Como Entorno De Desarrollo Integrado (Ide)}

¿El Dreamweaver, por qué?, bien, es necesario tener en cuenta que este editor es compatible con la mayoría de entornos de desarrollo web y móviles (phonegap)

\section{Figura 5}

Dreamweaver como un entorno de desarrollo integrado (IDE)

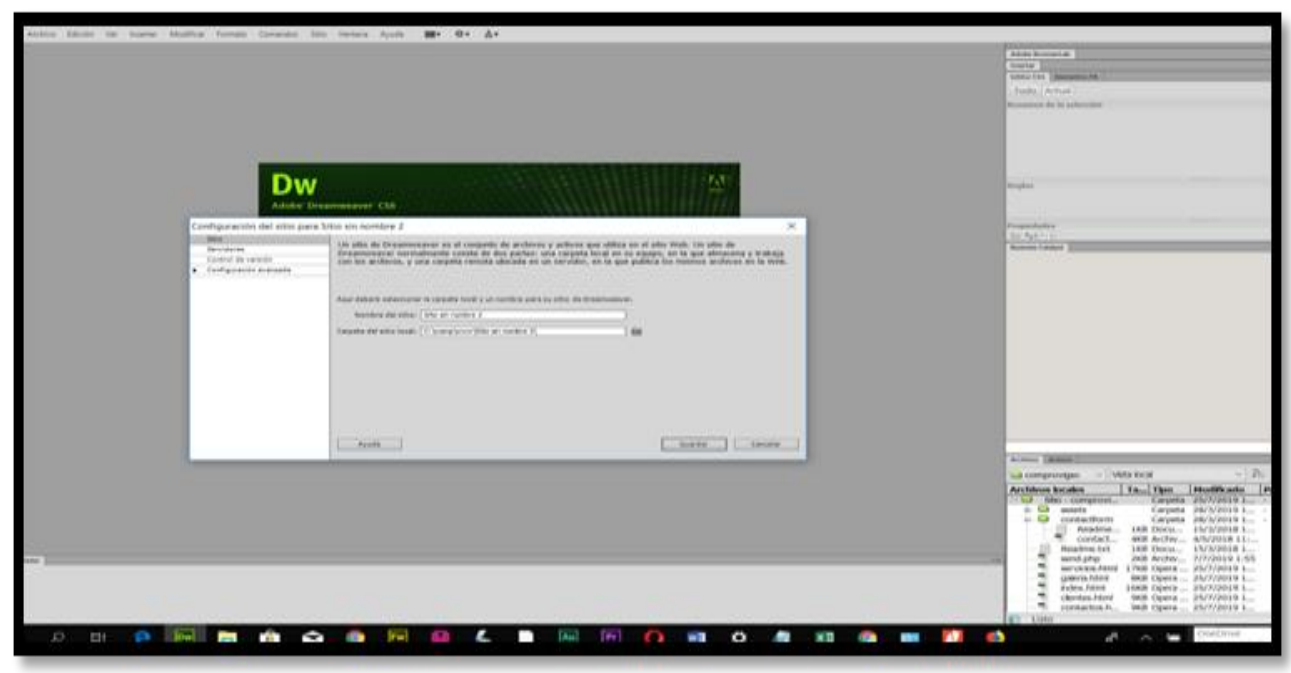

Fuente: Diego Bastidas (2019). 
Revista Arbitrada Interdisciplinaria KOINONIA

Año V. Vol V. N9. Enero - Junio 2020

Hecho el depósito de Ley: FA2016000010

ISSN: 2542-3088

FUNDACIÓN KOINONIA (F.K). Santa Ana de Coro. Venezuela.

Diego Javier Bastidas-Logroño; Oscar Omar Espíndola-Lara; Alex Darío Palma-Rivera

\section{Configuración Del Sitio Ftp(Protocolo De Transferencia De Archivos)}

Una vez abierto nuestro IDE es muy sencillo configurar, damos clic en la opción sitio, nuevo sitio, configuración ftp, ponemos el usuario y clave de nuestro hosting, en la opción prueba verificamos que la conexión sea exitosa.

\section{Figura 6}

Configuración del sitio con ftp

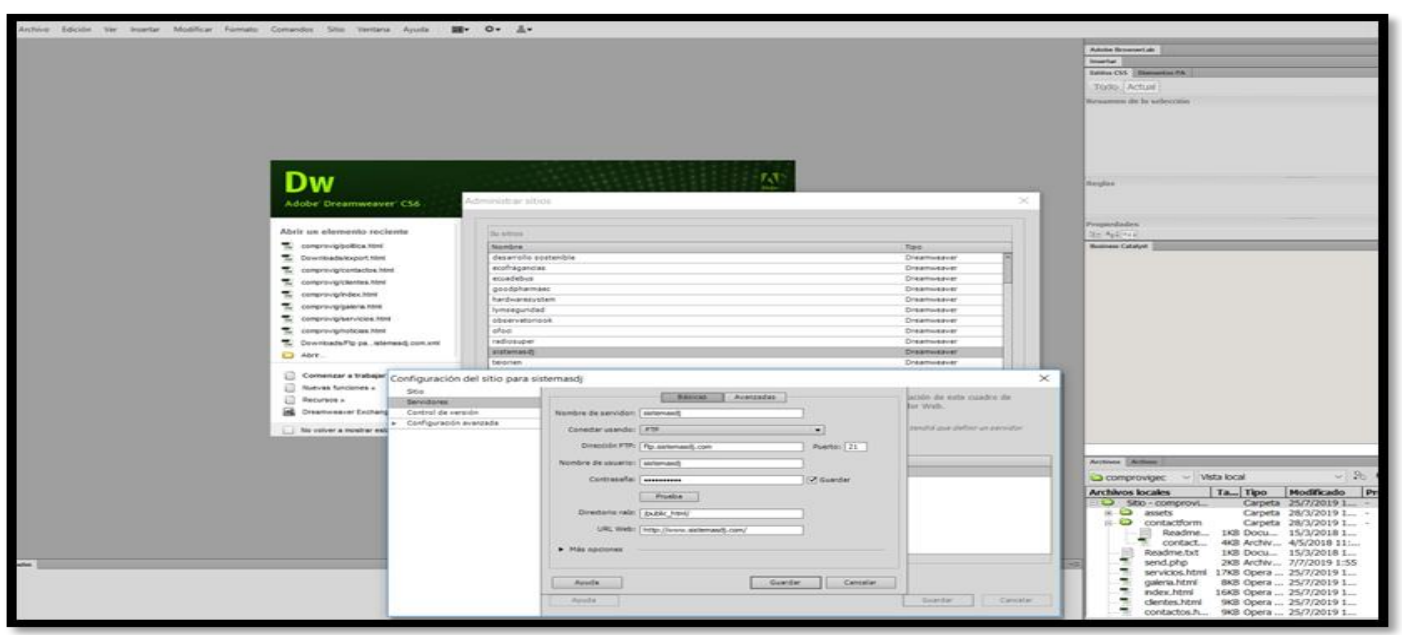

Fuente: Diego Bastidas (2019). 
Revista Arbitrada Interdisciplinaria KOINONIA

Año V. Vol V. N9. Enero - Junio 2020

Hecho el depósito de Ley: FA2016000010

ISSN: 2542-3088

FUNDACIÓN KOINONIA (F.K). Santa Ana de Coro. Venezuela.

Diego Javier Bastidas-Logroño; Oscar Omar Espíndola-Lara; Alex Darío Palma-Rivera

\section{CpanelWhm}

Los Planes RESELLER o revendedor como mi propuesta, es un panel de control a través del cual cada revendedor puede gestionar las cuentas de Hosting que genere para sus clientes.

\section{Figura 7}

Administración Host Web Básico

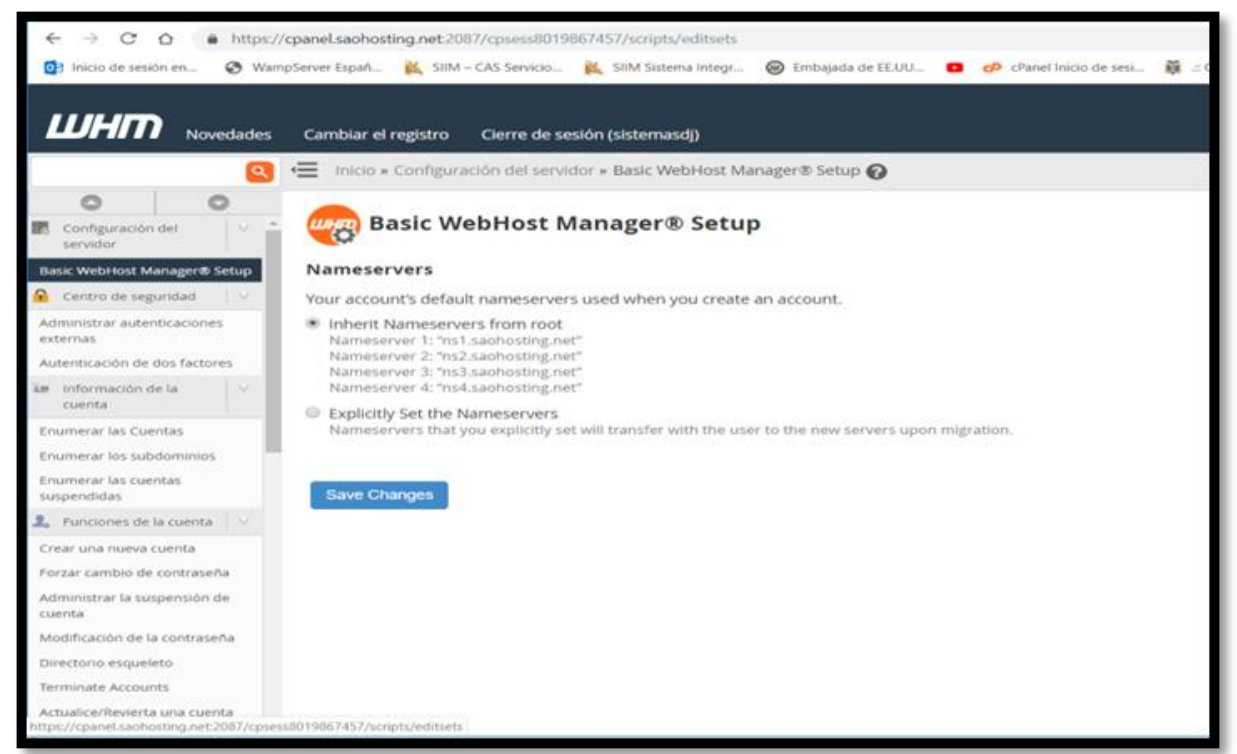

Fuente: Diego Bastidas (2019).

Así con tu espacio de hosting, tu propia empresa que al pagar un valor anual te convertirás en un revendedor de hosting, asignando cuotas de host a tus clientes dándoles unipersonalidad con un propio usuario y clave para ingresar a sus propios host de administración web. 
Revista Arbitrada Interdisciplinaria KOINONIA

Año V. Vol V. №9. Enero - Junio 2020

Hecho el depósito de Ley: FA2016000010

ISSN: 2542-3088

FUNDACIÓN KOINONIA (F.K). Santa Ana de Coro. Venezuela.

Diego Javier Bastidas-Logroño; Oscar Omar Espíndola-Lara; Alex Darío Palma-Rivera

\section{Figura 8}

Visualización de cuentas clientes con su propio host cpanel

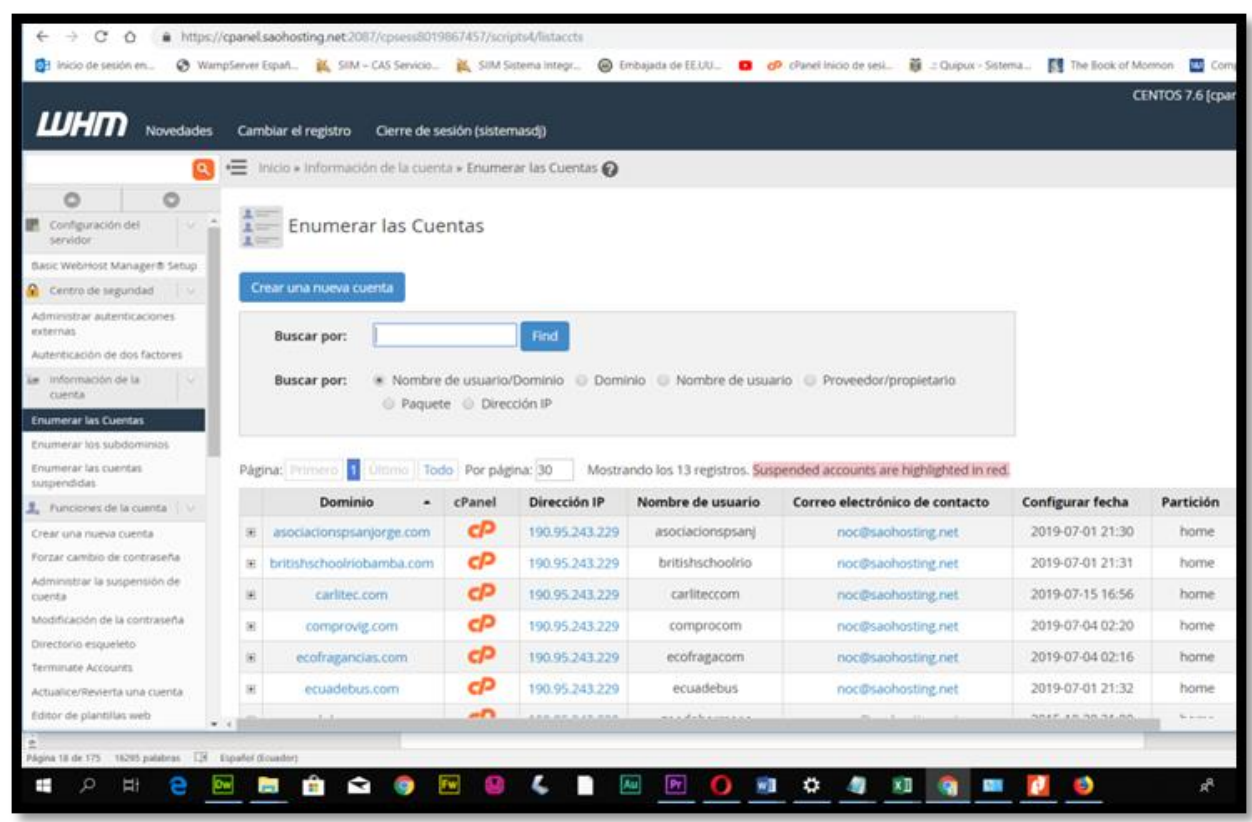

Fuente: Diego Bastidas (2019).

Entre los planes RESELLER o REVENDEDOR existen varios tomando como ejemplo la figura a continuación:

\section{Figura 9}

Ejemplo de planes reseller o revendedor

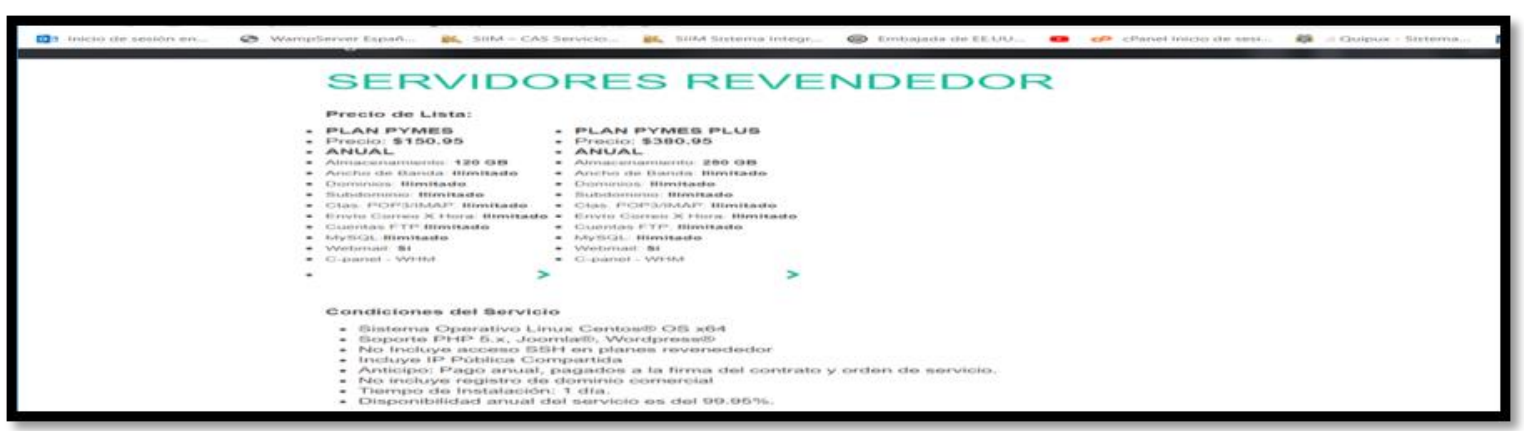

Fuente: https://www.saohosting.net/ 
Revista Arbitrada Interdisciplinaria KOINONIA

Año V. Vol V. N9. Enero - Junio 2020

Hecho el depósito de Ley: FA2016000010

ISSN: 2542-3088

FUNDACIÓN KOINONIA (F.K). Santa Ana de Coro. Venezuela.

Diego Javier Bastidas-Logroño; Oscar Omar Espíndola-Lara; Alex Darío Palma-Rivera

\section{Requerimientos Del Hardware}

La siguiente información representa los requerimientos mínimos del hardware.

Cpu

- Intel Pentium CoreDuo o superior

- $\mathrm{AMD} 64^{\star} 2$

- Memoria

- Mínimo $1 \mathrm{~GB}$

- Recomendado $2 \mathrm{~GB}$, para rapidez

\section{Disco Duro}

- 80 GB o superior recomendado recomendado. 
Revista Arbitrada Interdisciplinaria KOINONIA

Año V. Vol V. N9. Enero - Junio 2020

Hecho el depósito de Ley: FA2016000010

ISSN: 2542-3088

FUNDACIÓN KOINONIA (F.K). Santa Ana de Coro. Venezuela.

Diego Javier Bastidas-Logroño; Oscar Omar Espíndola-Lara; Alex Darío Palma-Rivera

\section{REFERENCIAS CONULTADAS}

Censo (2010). Fascículo Santo Domingo. [Santo Domingo fascicle]. Recuperado de https://n9.cl/2450s

Lauri B., Lauri P. Apache Definitive Guide, segunda edición, EEUU, O' Reily \& Associeties, Inc., 1999. [Guía definitiva de Apache, segunda edición, EE. UU., O 'Reily \& Asociaciones Inc 1999].

Maxwell Steve. Red Hat Linux Herramientas para la administración de Redes. [Red Hat Linux. Network administration tools].

Traducido del inglés por Gustavo Fonseca. Revisión Técnica Jamir Ávila, PrimeraEd. [Translated from English by Gustavo Fonseca. Technical Review Jamir Ávila, First Ed]. Bogotá, Mac Graw Hill, 2001.

Otto, M. \&Thornton, J. (2016). Bootstrap 3, el manual oficial. [Bootstrap 3, the official manual]. Recuperado de https://uniwebsidad.com/libros/bootstrap3 ?from=librosweb

Schenkt. Et, al. Administración de red Hat Linux. [Red Hat Linux Administration]. Traducido del Inglés porVuelapluma Primera ed. España, Prentice Hall. 2001.

Saohosting (2018). Cpanel herramientas. [Cpanel tools]. Recuperado de https://www.saohosting.net/

Universidad Pedagógica Experimental Libertador (2012). Manual para Realización de Trabajo de Grado Maestrías y Tesis Doctórales. [Manual for the Completion of Work on Master's Degrees and Doctoral Theses]. Edic. UPEL. Barquisimeto.

Widwell, D. Snell, J Y Kulchenko P. Programming Web Services with SOAP. [Programación de servicios web con SOAP]. Primera Ed. Estados Unidos, O' Reily \& Associetes Inc., 2001. 